\title{
On Under-determination in Cosmology
}

\author{
Jeremy Butterfield \\ Trinity College, Cambridge CB2 1TQ: jb56@cam.ac.uk \\ Published in Studies in History and Philosophy of Modern Physics 46 (2014), pp. 57-69 \\ Keywords: \\ philosophy of cosmology, under-determination, Manchak, cosmological principle, \\ inflation
}

5 July 2013

\begin{abstract}
I discuss how modern cosmology illustrates under-determination of theoretical hypotheses by data, in ways that are different from most philosophical discussions. I emphasize cosmology's concern with what data could in principle be collected by a single observer (Section 2); and I give a broadly sceptical discussion of cosmology's appeal to the cosmological principle as a way of breaking the under-determination (Section 3 ).

I confine most of the discussion to the history of the observable universe from about one second after the Big Bang, as described by the mainstream cosmological model: in effect, what cosmologists in the early 1970s dubbed the 'standard model', as elaborated since then. But in the closing Section 4, I broach some questions about times earlier than one second.
\end{abstract}




\section{Contents}

1 Introduction

1.1 Prospectus: two differences . . . . . . . . . . . . . . . . 3

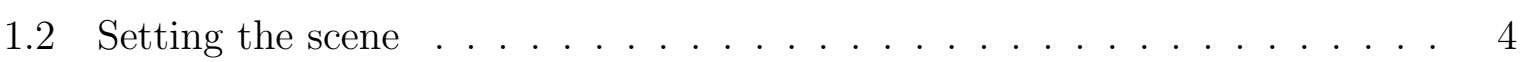

2 Observationally indistinguishable spacetimes 6

3 The cosmological principle to the rescue?

3.1 A lucky break . . . . . . . . . . . . . . . . . . . 10

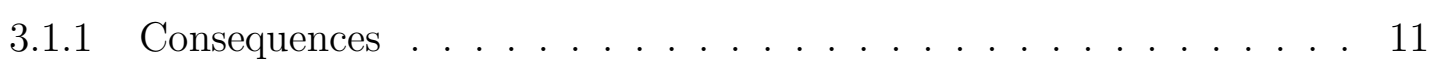

3.1 .2 Reasons ......................... . . . . . . . . . . .

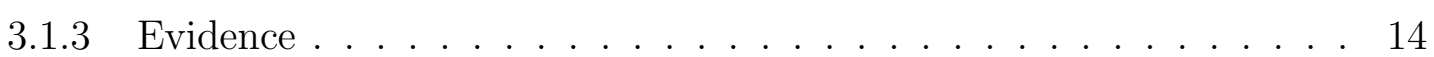

3.2 Beyond the evidence: through a glass darkly . . . . . . . . . . . . . 16

3.2.1 Strategies for justifying the CP . . . . . . . . . . . 16

3.2 .2 Inhomogeneities: doubts about the $\mathrm{CP} \ldots \ldots . . . . . . . .19$

4 Pressing the question 'Why?'

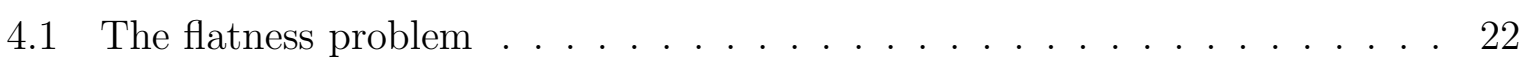

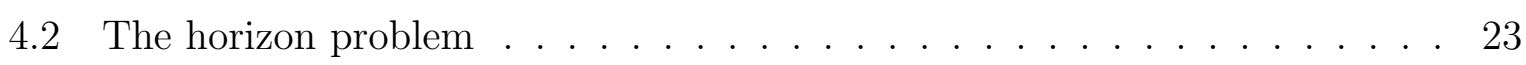

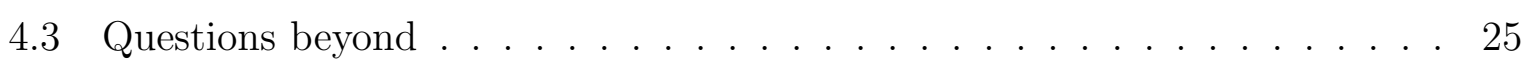

5 References $\quad \lcm{27}$ 


\section{Introduction}

\subsection{Prospectus: two differences}

The aim of this paper is to survey how modern cosmology illustrates the under-determination of theoretical hypotheses by data in ways that are different from most philosophical discussions. I will concentrate on two main differences, which I can introduce as follows.

The usual philosophical discussion of under-determination idealizes by assuming that all possible observations are 'given', even though of course they are spread throughout spacetime, and so never collected by a single scientific community. Then the discussion addresses whether a theory of tiny unobservable objects like electrons is under-determined; and relatedly, how good is our warrant for our accepted physical theories.

On the other hand, cosmology takes due cognizance of the difficulties both of observing parts of the universe that are very distant in time and space, and of collecting observations together at a single place and time. But (as one would expect): cosmology does not address the general philosophical debate about our warrant for our accepted theories, in particular our current theories of tiny unobservable objects. On the contrary, cosmology for the most part treats our current accepted physical theories as 'given'. For it is, like geology, a historical science: and as such, it aims to provide, not a general theory, but as detailed as possible a history of its topic - the universe 1

Here I say 'for the most part' because, although cosmology has in the last several decades gone from success to success in describing, in ever more detail, the history of the universe, using our accepted theories, especially general relativity and quantum theorynevertheless, this endeavour of course leads to unknown physics, especially at the very high energies in the very early universe. I myself would place the limit of justified confidence in applying our accepted theories at about one second after the Big Bang (a temperature of about $10^{10} \mathrm{~K}$ ); and I will confine my discussion to that and later times (lower energies) except for my last Section which poses, but does not pursue, some questions about earlier times 2

\footnotetext{
${ }^{1} \mathrm{My}$ usage here of 'historical science' is of course not meant to exclude prediction, the most striking current example being no doubt the universe's accelerating expansion. My usage just indicates the contrast between a general theory admitting many models (solutions, 'possible worlds') and a detailed description of a single model. Agreed, the usage of 'theory' and 'model' varies: for clarification, cf. below, especially (2) in Section 1.2 .

${ }^{2}$ Placing the limit at one second is cautious. Nowadays, it is common to take the boundary between known and speculative physics to be at about $10^{-11}$ seconds, before which the energies are too high for us to be confident that the standard model of particle physics applies. This time corresponds to the electro-weak phase transition. And even twenty years ago, a standard monograph was confident of its account from $10^{-2}$ seconds onwards (Kolb and Turner, 1990, p. 109). Cf. also Rees' remark that he is $99 \%$ confident of the account from one second onwards (2003, pp. 24, 31; 1997, p. 65, 174). For a philosophical entry into these details, cf. Zinkernagel (2002), Rugh and Zinkernagel (2009), Smeenk (2012, Section 6). My last Section will broach the idea of inflation, which is meant to have started much (logarithmically) earlier than $10^{-11}$ seconds, viz. at about $10^{-35}$ seconds: so I think caution about inflation is all the more justified. On the other hand, I also think that both this confrontation with unknown physics, and the under-determination which this paper is concerned with, are entirely compatible with scientific realism: I argue for this elsewhere (2012, Section 2).
} 
Thus I see two main differences. The first is that cosmology takes in to account the task of collecting at a single place and time all the possible data — which most philosophical discussions do not. When we add to this the supreme success of modern cosmology in describing the history of the universe from about one second after the Big Bang, we are led to the second difference. Namely: in cosmology, general theories are not, for the most part, the items that are under-determined. Rather what is under-determined is which model of our well-established theories is the correct one; i.e. which history of the universe as described by general relativity and quantum theory should we endorse. (Agreed, this difference is less crisp than the first, not least because the theory-model contrast is vague; cf. Section 1.2.)

The first of these differences is entrenched in a common jargon within cosmologywhich I will adopt. Thus cosmologists often use observable universe to mean (roughly speaking) 'the past light-cone of Earth-now, and all physical events within it, even microscopic (and so humanly unobservable) ones'; as against, say, 'all macroscopic or humanly observable events, anywhere in spacetime'. Combining this jargon with the second difference, we conclude: in cosmology, the issue of under-determination is often, not about our warrant for our general theories, but about whether the data about the observable universe determine a single model of these theories as being correct.

I will proceed in three main stages. In Section 2, I will discuss recent results of Manchak $(2009,2011)$ : their gist will be that according to general relativity, the correct model is endemically under-determined by data, even ideal data, about the observable universe (i.e. past light-cone of an observer). This predicament prompts the question whether we can justify some principle that cuts down the dismaying variety of models. The cosmological principle is probably the strongest and best known candidate for such a principle. So in Section 3, I survey how we might go about justifying it, by general arguments and-or by empirical evidence. My verdict will be mostly negative: although the cosmological principle holds up well as an idealized description of the observable universe, i.e. our past light-cone, it is very hard to justify for the universe as a whole. I will end, in Section 4, by posing (but not pursuing) some questions that arise, including questions about times earlier than one second after the Big Bang (and correspondingly higher energies). But first: I should set the scene, by briefly discussing some other aspects of under-determination in cosmology.

\subsection{Setting the scene}

In cosmology, under-determination is not always understood in the way I have sketched. Hence I said 'often', rather than 'always', when I reported how cosmologists use 'observable universe', and when I said that under-determination is about whether data about the observable universe determine a single model.

As I see matters, there are two other main construals of 'observable universe', and-or other relevant terms like 'under-determination'. It will help to state them explicitly: not least because they are both close to common philosophical concerns. The first brings us closer to philosophers' usual theory-observation distinction. The second registers the 
variety of uses of 'theory' and 'model'.

(1): 'Observable' in the light of decoupling: Thus cosmologists also use 'observable universe' more narrowly, reflecting their concern with what can be ascertained by observation. The last several decades have of course seen great advances in cosmological observation: both in the types of radiation (parts of the electromagnetic spectrum) and of matter observed, and in observations' precision and depth; ('depth' in time as well as space: cf. e.g. Longair (2003, Chapter 18; 2006 Chapters 7, 11, 13). The most famous advance has been the cosmic microwave background radiation (predicted in 1950 and discovered in 1965) which dates from the decoupling time, about 400,000 years after the Big Bang: and which is nowadays our single most important line of evidence for the Big Bang, and about the early universe - witness the data about it from the COBE, WMAP and Planck projects. But we cannot now directly observe by any electromagnetic means any earlier events, since until the decoupling time the universe was opaque to radiation. So until and unless we observe e.g. gravitational radiation from such earlier times, cosmologists often take the phrase 'observable universe' to mean: 'the events in the past light-cone of Earth-now, and to the future of the decoupling time, that our instruments can detect'. With this sort of usage, the issue of under-determination of theory by observation in cosmology becomes closer to the familiar philosophical issue of the fallibility of inference from the observed to the unobserved.

(2): 'Theory' vs. 'model': I said that cosmology treats our general theories, such as general relativity and quantum theory, as 'given'; and using them, it has provided a very successful, ever more detailed, history of the universe. And I qualified this, adding 'for the most part', only by registering our ignorance of the very early universe. (This qualification is similar to the shift from my first, adopted, sense of 'observable universe', to that in (1) above.)

Broadly speaking, that is true enough. Consider how the overall thermal history of the observable universe has been well established since about 1970. We know that about 13 billion years ago, there was an extremely hot and dense 'fireball' in which light nuclei (like hydrogen and helium) were synthesized, according to well-understood nuclear physics; it cooled and expanded, with the details being accurately described by quantum theory, thermodynamics and general relativity; later, gravitational clustering led to galaxies and stars, in which still more elements were synthesized 3

\footnotetext{
${ }^{3}$ Thus authoritative textbook descriptions of this thermal history, written over the last forty years, largely agree with each other. Cf. for example: Sciama (1971: Chapters 8, 12-14), Weinberg (1972, Chapter 15.6, pp. 528-545), Wald (1984, pp. 107-117), Barrow and Tipler (1988, pp. 367-408, Sections 6.1-6.7), Lawrie (1990, pp. 315-326), Longair (2006, 394-399), Weinberg (2008, pp. 101-113, 149-173; Sections 2.1, 2.2, 3.1, 3.2). For fine popular accounts, cf. Silk (1989, Chapters 6 to 8), Rowan-Robinson (1999, Chapter 5), Silk (2006: pp. 112-128).

Besides, the thermal history of the observable universe is by no means the only over-arching cosmological claim that is now firmly established. The theory of stellar structure and evolution is another example, for which one could similarly cite authoritative descriptions over several decades largely agreeing with each other.

This consensus is reflected in cosmologists' jargon: what philosophers might call 'the Big Bang theory' was dubbed by cosmologists 'the standard model', already in the early 1970s (Weinberg (1972, p. 469), Misner et al. (1973, p. 763)). At that time, the honorific name reflected its recent confirmation by the
} 
But I should add two other qualifications. Both are independent of issues about the early universe; and both give us a warning that the usages of the words 'theory' and 'model' are very variable. (Thanks to C. Beisbart, C.Smeenk, W. Stoeger and H. Zinkernagel for emphasizing these to me.)

(a): First, in this standard cosmological account (called 'the standard model': cf. footnote 3), there remain major causal and structural factors that are not understood: such as the nature of dark matter and dark energy, and the process of galaxy formation. So there are many 'versions' of 'today's standard model'. In assessing these versions, there is much to do, both theoretically and observationally. But as regards the philosophical issue of under-determination, the situation seems to me to be 'business as usual'. (But we must beware the varying jargons: as I have just expressed it, we here face 'under-determination of versions by presently available data'.)

(b) Second: this standard cosmological account, 'the standard model', is not established beyond doubt: there is respectable dissent. As we will see in more detail in Section 3, this account incorporates spatial homogeneity (by assuming the cosmological principle); but there are rival inhomogeneous models which match the presently available data without invoking dark energy (cf. e.g. Sarkar (2007), Nadathur et al (2011)). So here again, we have 'business as usual': a philosopher would speak of a dispute over - or underdetermination by presently available data of - theories; and there is good reason to hope the dispute will be resolved by observations over the next decade or two 4

\section{Observationally indistinguishable spacetimes}

I turn to presenting the endemic under-determination of cosmological models in general relativity, by reporting the theorems of Manchak (2009, 2011); (which build on ideas and results of Glymour (1977) and Malament (1977)). Roughly, the theorems say that in almost every spacetime obeying general relativity, no observer, however long they live, could accumulate enough observations to exclude their being in another very different spacetime.

I begin with some standard notation and jargon. (1): In general relativity, a model is given by a spacetime $(M, g)$ consisting of a four-dimensional manifold $M$ of spacetime

discovery of the cosmic background radiation: nowadays, having stood up to many more observational tests, it deserves the name all the more.

${ }^{4}$ For more general perspectives on inhomogeneous (and anisotropic) models as tenable rivals to the cosmological principle, cf. e.g. MacCallum (1979), Ellis (2007, Section 4.2.2, pp. 1223-1227; 2011, Section 4, pp. 11-15).

Beware of another variation in jargon. Cosmologists also use 'model' for a description of (the main features of) a single history, for example in the name 'the concordance $\Lambda \mathrm{CDM}$ model', where $\Lambda$ refers to the dark energy, and 'CDM' refers to 'cold dark matter'. This model is the best fit of the standard model (an FRW metric as in Section 3.1.1 and so on) to all observations made so far, and is accordingly very specific about the values of cosmological parameters. It says, in short: 73 per cent dark energy, 27 per cent matter (split between baryonic and dark matter, with about six times more dark matter than baryonic matter), geometrically flat (with the density parameter $\Omega$ very close to 1 ) and the Hubble constant $H_{0}$ about $70 \mathrm{~km} / \mathrm{sec} / \mathrm{Mpc}$. But to return to (b): I stress that although this concordance model represents the current consensus, there are respectable rivals. 
points, equipped with a Lorentzian metric tensor $g$.

(2) A region of one model, $U \subset M$, is isometric to another region of another model, $U^{\prime} \subset M^{\prime}$, if there is a diffeomorphism $d: U \rightarrow U^{\prime}$ that carries $M$ 's metric $g$ as restricted to $U$ into $M^{\prime}$ 's metric $g^{\prime}$ as restricted to $U^{\prime}$. This means full knowledge of all the metrical relations between points in $U$ could not exclude one's being instead in $U^{\prime}$.

(3): Of course, one usually knows much less than everything about metrical relations, and more than nothing about spacetime's matter and radiation content: which so far, seems culpably unrepresented in our notion, $(M, g)$, of a cosmological model. But the theorems and discussion can probably be adapted to include facts about matter and radiation; (Malament 1977, pp. 74-76) 5

(4): We formally define the past light-cone of (an observer at a) spacetime point $p \in M$, as follows. (i): For points $p, q \in M: q$ causally precedes $p$ if there is a future-directed curve in $M$ from $q$ to $p$ whose tangent vector at every point is timelike or lightlike. (ii): The causal past of $p$ is the set of points that causally precede it: $J^{-}(p):=\{q$ : $q$ causally precedes $p\}$. (One similarly defines the causal future $J^{+}(p)$.) But it turns out, for technical reasons, to be easier to work with the interior of $p$ 's past light-cone, i.e. the points connectible to $p$ by signals travelling slower than light. Formally, these are the points connected to $p$ by a future-directed curve whose tangent vector at every point is timelike: dubbed the chronological past and written $I^{-}(p)$.

Summing up this notation and jargon: Manchak's theorems will show that an ideal observer at $p \in M$ who knows the full metric structure of $I^{-}(p)$ cannot know much about the global structure of her spacetime, since many different spacetimes, with widely varying global properties, have a region isometric to $I^{-}(p)$. More precisely: let us say that a spacetime $(M, g)$ is observationally indistinguishable from $\left(M^{\prime}, g^{\prime}\right)$ iff for all points $p \in M$, there is a point $p^{\prime} \in M^{\prime}$ such that $I^{-}(p)$ and $I^{-}\left(p^{\prime}\right)$ are isometric. (The fact that this notion is asymmetric will not matter.) Then the gist of the theorems is that almost every spacetime is observationally indistinguishable from another, i.e. a non-isometric spacetime.

So much for the gist. The precise theorems incorporate (i) a mild limitation, and (ii) two significant generalizations; as follows.

(i): The theorems set aside spacetimes $(M, g)$ that are causally bizarre in the sense that there is a point $p \in M$ such that $I^{-}(p)=M$. (This strong condition implies various causal pathologies, in particular that there are closed timelike curves.)

(ii): But the theorems accommodate any further conditions you might wish to put on spacetimes, provided they are local, in the sense that any two spacetimes $(M, g)$ and $\left(M^{\prime}, g^{\prime}\right)$ that are locally isometric (i.e. any $p \in M$ is in a neighborhood $U \subset M$ that is isometric to a neighborhood $U^{\prime} \subset M^{\prime}$, and vice versa) either both satisfy the local condition, or both violate it. (So this generalizes the point in (3) above that the theorem accommodates having observations of facts about matter and radiation.)

The theorems also veto an observer's ascertaining some global properties of her spacetime. Manchak lists four such properties (2011, pp. 413-414). Three are 'good causal

\footnotetext{
${ }^{5}$ Besides, Einstein's equations for general relativity imply that the metric $g$ mathematically determines another tensor $T$, the stress-energy tensor, which encodes some main facts about matter and radiation. Hence the widespread practice of defining a model by a spacetime $(M, g)$ rather than a triple $(M, g, T)$.
} 
behaviour' properties: viz. that the spacetime be globally hyperbolic, inextendible and hole-free. We will not need their definitions. But the fourth property will be crucial to the cosmological principle (cf. Section 3.1). It is spatial isotropy: there being, at every spacetime point, no preferred spatial direction. Thus the theorems will veto an observer's ascertaining these properties: given a spacetime $(M, g)$ with any or all of these properties, there is an observationally indistinguishable spacetime with none of them.

Thus Manchak's theorems (2009, Theorem, p. 55; 2011, Proposition 2) say: Let $(M, g)$ be a spacetime that is not causally bizarre, and that satisfies any set $\Gamma$ of local conditions. Then there is a non-isometric spacetime $\left(M^{\prime}, g^{\prime}\right)$ such that:

(a): $\left(M^{\prime}, g^{\prime}\right)$ satisfies $\Gamma$, but has none of the four listed global properties;

(b): $(M, g)$ is observationally indistinguishable from $\left(M^{\prime}, g^{\prime}\right)$.

The proof is short, and conceptually simple. It involves a stupendous 'cut-and-paste' construction, using (copies of) patches of the given spacetime $(M, g)$ to define the other one $\left(M^{\prime}, g^{\prime}\right)$-but stringing the patches out separately from one another, like shirts hanging on a clothes-line, so that $\left(M^{\prime}, g^{\prime}\right)$ is not isometric to $(M, g)$. The idea of the clothesline construction goes back to Malament: whose discussion also emphasizes that global properties which $(M, g)$ might have can fail to hold in $\left(M^{\prime}, g^{\prime}\right)(1977, \mathrm{pp}$. 70-74).

I have already noted that the theorems, despite their apparent formulation, allow for observations of matter and radiation. I should also note three other strengths.

(i): One might consider observations collected by a single immortal observer, or by an eternal dynasty of observers with each generation bequeathing its observations to the next. But I have here stated the theorems in terms of the past of a single point $p$, and so apparently made the threat of under-determination weaker. For even if $p$ is "late" in the universe, and has a vast past, might not later data break the under-determination? In fact, the threat cannot be allayed in this way: Malament (1977, pp. 63, 66-68).

(ii): One might object that the theorems are too idealized. In particular, how could an observer ever pin down the geometry of $I^{-}(p)$ ? But there are impressive, albeit again idealized, theorems saying that this is possible (Ellis (1980), Ellis et al. (1985, especially Section 12, pp. 377-390)). The idea is that assuming general relativity and some technical assumptions, observations of a suitable set of 'standard objects' of known size, mass and luminosity are necessary and sufficient to determine the geometry of $I^{-}(p)$.

(iii): The definition of observational indistinguishability, and thereby Manchak's theorems, are in a sense stronger than they need to be, in order to show under-determination. For surely, under-determination does not require that there be one spacetime $\left(M^{\prime}, g^{\prime}\right)$ such that, for all points $p \in M$, there is a point $p^{\prime} \in M^{\prime}$ with a chronological past isometric to that of $p$. Rather, it at most requires that for each $p \in M$, there exists a spacetime $M^{\prime}$ containing a point $p^{\prime}$ whose chronological past is isometric to that of $p$. That is: the spacetime $M^{\prime}$ can depend on the choice of $p \in M$. (Thanks to a referee for this point.)

One might worry about the scientific significance of the theorems: isn't the 'cut-andpaste' construction 'unphysical'? But that vague word covers several possible misgivings. One is that the theorems entirely concern classical general relativity, which we have every reason to believe fails for the extreme conditions in the very early universe and black holes. But this points beyond standard cosmology - and so beyond this paper. 
Within classical general relativity, the theorems are not so easily dismissed. That we define a spacetime model by a cut-and-paste construction is no evidence at all that the features it exhibits are not generic among general relativity's models. (I will return to the idea of generic states, in Sections 3.2.1 and 4.) And as to the specific four global properties that Manchak shows to be impossible to ascertain observationally: he reviews various authors' rationales for requiring one or more of these properties, concluding sceptically that none of these rationales are convincing (2011, Sections 5 and 6, p. 415f.). I also commend Norton's similarly judicious assessment (2011, especially Sections 5 and 6), and his sceptical conclusion that we are at a loss to justify the inductive inferences, which favour 'reasonable' spacetimes over 'gerry-mandered' observationally indistinguishable alternatives, that we intuitively endorse 6

The next Section takes up the discussion of one of these properties, spatial isotropy, in the context of cosmology's standard model. So the question will be whether, despite Manchak's and Norton's doubts, we can cut down the great variety of models allowed by the Malament-Manchak theorems by appealing to a principle of uniformity or simplicitythe cosmological principle.

\section{The cosmological principle to the rescue?}

The cosmological principle (CP) is one of several principles that have been appealed to since the mid-twentieth century in order to make cosmological models simple enough to be tractable. Indeed, the CP is the strongest and probably best known of them. Roughly speaking, it says that at any time, the universe is approximately the same at all spatial positions, and in all directions. Evidently, that is a logically strong assertion, which can be expected to reduce the threat of under-determination - but which would also need a lot of justifying.

Broadly speaking, this Section describes how the CP is what is colloquially called a 'lucky break', i.e. a piece of good fortune - so far. That is: in Section 3.1, I will report that $\mathrm{CP}$ does greatly reduce under-determination (in physicists' jargon: constrain cosmological models), and that observations of many kinds suggest CP holds good, on sufficiently large scales, of the observable universe. But Section 3.2 will urge that we have much less warrant for believing it of the entire universe. So, broadly speaking, Section 3.1 s discussion will be upbeat, and will mention some of the great observational and theoretical achievements of modern cosmology. But Section 3.2 s discussion will be downbeat. It will bring out how much we have yet to understand; including about issues like what would be an appropriate probability measure over initial conditions, which lead into controversies about the very early universe (i.e. times logarithmically much earlier than one second). It also meshes with Manchak's result (Section 2) that no observer can ascertain observationally that her spacetime is everywhere spatially isotropic; (for spatial isotropy implies the spatial homogeneity that the CP also asserts: cf. Section 3.1.2).

\footnotetext{
${ }^{6}$ Agreed: there may be some constructions of spacetimes that we can justify condemning as contrived. Thus Magnus (2005, Section 6) makes a good case that we should condemn a spacetime that repeats initial data (maybe many, even infinitely many, times) by disidentifying the initial data in a given spacetime.
} 
Given the CP's central role in cosmology, both theoretically and observationally (and ever since it was formulated, in 1935), this Section will be dismayingly brief. Apart from lack of space, my excuse is that the CP's central role has prompted a good deal of attention to it, over the decades, in the philosophical literature; including comparison with other such principles, such as the 'Copernican principle' (e.g. Ellis 1975, pp. 252-257), and 'Weyl's principle' (e.g. Rugh and Zinkernagel 2011). I especially recommend recent work by Beisbart (2009, 2012). His (2009) focusses on the CP's breaking under-determination, and what follows is but a glimpse of his treatment. (Cf. also Beisbart and Jung (2006); for a brief overview by a maestro, cf. Ellis (2007, p. 1223-127, Section 4.2.2).

\subsection{A lucky break}

The CP says that on sufficiently large scales, the universe and its material contents are (a) spatially homogeneous and (b) spatially isotropic about every spacetime point. Roughly speaking, this means that, although both spatial geometry and the matter and radiation content of space can change over time, there is a foliation of the spacetime into spacelike hypersurfaces, on any one of which: (a) at any two points, the state (of metric, matter, radiation etc.) is the same, and (b) at any point, all spatial directions are equivalent. (For precise definitions of both (a) and (b), cf. e.g. Weinberg (1972, pp. 378-381, 409-413), Wald (1984, pp. 92-94).)

In this principle, the idea that space itself is homogeneous and isotropic is very familiar from Newton's physics. Beyond this, the principle expresses two ideas.

(1): The first is the material contents being required to be homogeneous and isotropic. Here the qualification 'on sufficiently large scales' is crucial, since these material contents are evidently inhomogeneous and anisotropic on most observed scales. Matter is clumped together in bodies separated by apparently empty space. And at various scales, various directions are preferred; e.g. up-down, at human scales on Earth; and to/from the Sun, at solar-system scales. A priori, it is unclear whether local perturbations in matter density are small enough in size, and-or similar enough from one spatial region to another, that averaging over large enough length-scales will "wash them out". In fact, there is an elementary heuristic argument, based on the Newtonian approximation, that this is so 7

\footnotetext{
${ }^{7}$ In Newtonian theory, a deviation $\delta \rho$, over a length scale $L$, from the Universe's mean density $\rho$ is related to the perturbation $\delta \Phi$ in the gravitational potential $\Phi$ by: $\delta \Phi \sim \delta \rho L^{2}$. Applying this to a pressureless FRW Universe at a late cosmic time $t$ (cf. Section 3.1.1), the relative size of the metric perturbations is given by

$$
\delta \Phi / \Phi \sim(\delta \rho / \rho)(L / c t)^{2} .
$$

The CP requires that $\delta \Phi / \Phi<<1$. But eq. 3.1 shows that this allows $\delta \rho / \rho$ to be $>>1$, provided that $L<<$ ct. Indeed, a macroscopic object e.g. a person provides a $\delta \rho / \rho \sim 10^{30}$ over-density compared with the Universe's mean density, on a length scale $L \sim 1$ metre. But at the present epoch, $c t \sim 10^{25}$ metres, so that $\delta \Phi / \Phi \sim 10^{30} \times 10^{-50} \sim 10^{-20}$. Thanks to John Barrow for explaining this to me. Cf. his (1993, pp. 121-122), which also explains how: (i) the temperature fluctuations $\delta T / T$ in the cosmic background radiation give a direct probe of metric perturbations (indeed: $\delta T / T \sim \delta \Phi / \Phi)$ and are so tiny $\left(10^{-5}\right)$ as to strongly support the CP; (ii) eq. 3.1, together with $\delta \rho / \rho \sim L^{-2}$ (postulated by inflation), explains the scale-independence of metric perturbations.
} 
So here, the broad physical issue is: how large a scale must we consider in order for the CP to be tenable? Section 3.1.3 will report the present-day answer, and Section 3.2 .2 will briefly discuss the significance of inhomogeneities.

The broad philosophical issue is the nature and justification of idealizations. I will not pursue this, but I recommend: for general discussion, McMullin (1985), and for cosmology Ellis (1991, pp. 561-568; 2011 Sections 2,3, pp. 3-11) and Beisbart (2012, Section 4). For example, Beisbart surveys two approaches to understanding the phrase 'sufficiently large scales' in the CP. One is coarse-graining: roughly speaking, this is taking averages of a quantity such as density or velocity over larger and larger volumes so that the 'clumps' get washed out. The other is statistical: roughly speaking, this is taking the actual clumpy distribution of matter to be a realization of a stochastic process that is homogeneous and isotropic in the statistical sense that its probability distributions are invariant under translations and rotations. These approaches obviously raise interesting conceptual questions, such as how to understand probability applied to the universe- but I cannot pursue them.

(2): The CP's second idea is the allowance that the geometry and material contents of the universe can change over time. This allowance has been familiar since the midtwentieth century, thanks to Hubble's discovery of the expansion of the universe, and the formulation of Big Bang models of its evolution by theoreticians such as Friedmann, Lemaitre and Gamow. But it is worth recalling how radical this allowance seemed at first. Notoriously, Einstein's initial motivation in 1917 for introducing a cosmological constant $\Lambda$ into general relativity was to find a cosmological model that would be constant in time. ( $\Lambda$ represented a repulsive force acting at large distances which in Einstein's model exactly balanced the implosive tendency of gravity.) And the steady-state theory vetoed this allowance by postulating a 'perfect $\mathrm{CP}^{2}$ ' which required homogeneity in time, i.e. constancy, as well as in space. (For the history, cf. Kragh (1996, p. 182f.), Longair (2006, Chapter 12).)

The CP is a 'lucky break', in three ways, which I shall discuss in turn.

(1): It has strong and mathematically tractable, indeed elegant, consequences for the spacetime metric; it thereby promises to allay the threat of under-determination (Section 3.1.1).

(2): There are impressive mathematical theorems about its relation to other principles, and about how observational evidence could support it (Section 3.1.2).

(3): As I mentioned in the preamble to Section 3, for the observable universe, it is well-confirmed (Section 3.1.3).

\subsubsection{Consequences}

CP implies that (on sufficiently large scales) the spacetime metric ( $g$ in the notation of Section 2) is everywhere of a very simple form: the now-famous Friedmann-RobertsonWalker (FRW) form. This means that:

(i) there is a cosmic time-function $t$ whose simultaneity surfaces (also known as 'spatial slices': spacetime points with the same value of $t$ ) foliate the spacetime; 
(ii) the entire spacetime geometry is described by just:

(a): an integer $k=0$ or +1 or -1 , representing the curvature of the spatial slices ( $k=0$ means euclidean geometry, $k=+1$ means a geometry like surface of a sphere etc.); and

(b): a function $R(t)$ that represents the scale-factor, or 'radius', of the universe at cosmic time $t$.

The evolution of $R(t)$ depends not only on $k$, but on the type of matter and radiation (and so on how they interact) and on the value of the cosmological constant $\Lambda$. Thus by choosing various types of matter and radiation, and values of $k$ and $\Lambda$, one can write down some dozen or two dozen simple cosmological models. Some are stationary, some expand forever, some expand and then re-collapse; some have a flat spatial geometry $(k=0)$, some a curved one 8

Of course, in the forty years 1925-1965, before the rise of cosmology as an observational science with a great variety of precise observational techniques (cf. Section 3.1.3), the expanding models in this class were regarded as giving only a broadest-brush description of the evolution of the universe. And of course, only for times well after any earliest epochs: in particular, well after any 'primeval atom' or 'primeval fireball' of the type advocated by Lemaitre and Gamow. Nevertheless, these models married Hubble's great discovery that the galaxies were receding from us - and the associated 'Copernican' idea that this observation should be 'typical', in that any observer would see galaxies recedingto our best theory of gravity, Einstein's general relativity. Accordingly, they were much investigated: and to the extent that observation allowed, their parameters (of the rate of expansion, the age of the universe etc.) were estimated. (For the history, cf. Kragh (1996, especially Chapter 6), Longair (2008, Chapters 6, 13).) For our philosophical topic of under-determination, the moral is clear: for these models to be predictive enough to confront observations - even the meagre observations of the mid-twentieth century - their incorporating CP was crucial.

\subsubsection{Reasons}

So much for the CP's consequences. What reasons can be given for believing it? Section 3.1.3 will report the favourable evidence, in the straightforward inductive sense, rooted in observation. Here I will briefly list some theoretical reasons. By this, I mean theorems that in effect reduce what we would be required to observe in order to get evidence supporting the CP.9

\footnotetext{
${ }^{8}$ For details of this class of possible universes, in authoritative textbooks (deliberately different from those in footnote 3), cf. Misner et al. (1973, Chapter 27), Hawking and Ellis (1973, pp. 124-148), Rindler (1977, Chapter 9), Liddle (2003, Chapters 4, 5, 15.1), Rowan-Robinson (2004, Chapter 4); or with a view to philosophy, Ellis (2007, pp. 1185-1191, Section 2.1). For a masterly popular account, cf. Barrow (2011, Chapter 3).

There are also important subtleties about how CP yields features (i) and (ii). Thus Rugh and Zinkernagel (2011, Section 3) emphasize that for CP to secure the cosmic time-function in (i), one needs to presuppose the congruence of timelike curves representing the mean motion of matter ('Weyl's principle').

${ }^{9}$ Since these theorems are about necessary and-or sufficient conditions for homogeneity and-or isotropy, they are of a similar ilk to results about the consequences of the CP. Among those consequences, Section
} 
This means I will set aside other, more rationalist, grounds for believing the CP, that over the decades, various authors have adduced; for example, arguments based on the 'Copernican' belief that our observations should be 'typical'. Stating and assessing such grounds and arguments has long been a central theme in philosophy of cosmology. But broadly speaking, recent assessments find these grounds and arguments unpersuasive; for example, the 'Copernican typicality' argument, despite its appealing 'modesty', is unconvincing - for we must admit there could be selection effects. I concur with these recent sceptical assessments, and so set aside these grounds and arguments. For details, I recommend Balashov (2009), Beisbart and Jung (2006, Sections 4, 5), Beisbart (2009, pp. 188-189, 193-199), Ellis (1975, especially pp. 250-257), and Norton (2011).

I will first discuss purely geometrical theorems relating the homogeneity and isotropy of the metric to each other, and to other geometrical conditions; (then I will discuss theorems about physical fields). As examples, here are three theorems.

(i): Homogeneity together with isotropy about a given point implies isotropy about every point on the manifold (e.g. Weinberg (1972, p. 379)

(ii): If a spacetime is spherically symmetric (as defined by e.g. Wald (1984, p. 120)) about every point, then it is homogeneous (Hawking and Ellis (1973, p. 135, 369). Combining this with (i), we infer: if a spacetime is spherically symmetric about every point, and isotropic about some point, then it is homogeneous and isotropic everywhere. That is: the CP holds.

(iii): Isotropy about every point implies homogeneity (Weinberg (1972, p. 379)). The idea is that isotropy at a point means that it has a neighbourhood on which a spatial rotation, mapping points of the neighbourhood among themselves, preserves the metric structure; and if this is true at every point, then the metric is the same everywhere. (Or more visually: think of connecting any two points by a sequence of rotations about suitably chosen other points, so that isotropy applied at these other points implies homogeneity.)

Summing up: the gist of these theorems is that we could justify the CP if we could justify spherical symmetry about every point, and-or isotropy about every point.

Second, there are theorems relating the metric's spatial homogeneity and isotropy to physical fields. Perhaps the most famous is the Ehlers-Geren-Sachs theorem (Ehlers et al. 1968). It says, roughly speaking, that for an expanding spacetime with pressureless matter and radiation: if the radiation is spatially isotropic about each spacetime point, then the CP holds, i.e. the metric is spatially homogeneous and isotropic and therefore is FRW. This theorem is remarkable for two reasons.

(i): One might argue for the theorem's antecedent by combining the 'Copernican principle' mentioned above (that our observations should be typical) with the fact that the cosmic background radiation has indeed been observed to be isotropic about what amounts, for cosmological scales, to being 'one' spacetime point: viz. Earth-1965, or with much better precision, Earth-2012.

(ii): The theorem is stable. More precisely: Stoeger et al. (1995) show that almost

3.1.1 focussed only on the FRW metric. But an exposition surely should emphasize the metric; and since these theorems focus on observational requirements, it is worth separating them out from the general idea of the CP's consequences. 
isotropic radiation measurements imply that the metric is almost in the FRW form. (Cf. the mention of idealization in the preamble to Section 3.1.)

Finally, there are various theorems that avoid assumptions about the physical situation at all (or even many) spacetime points; and so whose empirical application in cosmology can be less reliant on the Copernican principle a la (i) just above. I thank G. Ellis for pointing out to me a remarkable recent theorem of this kind. Clifton et al. (2011) show that by observing the cosmic background radiation for an extended period of time, or by observing scatterings of this radiation, a single observer can in principle conclude that their past light-cone's metric is homogeneous and isotropic. (The theorem exploits an effect, the kinematic Sunyaev-Zel'dovich effect, whereby an anisotropy of the radiation, as seen by an object that scatters the radiation towards us, causes a distortion in the radiation's spectrum as seen by us.)

\subsubsection{Evidence}

I turn to assessing the observational evidence for the CP. Recall that all our observational evidence comes from our past light-cone: which, following cosmologists' practice, I have called 'the observable universe'. In the notation of Section 2, all our evidence comes from $J^{-}$(Earth-now). So we should of course distinguish two sorts of evidence, or inductive inference:

(i) evidence that, or inference to the proposition that: CP holds for our past lightcone; and

(ii) evidence that, or inference to the proposition that: $\mathrm{CP}$ holds for the universe beyond our past light-cone.

Topic (ii) can be addressed in various ways: as we will see in Section 3.2. Here I just consider (i). As I said, this will be a matter of 'good news': a lucky break. I postpone the 'bad news' till Section 3.2 ,

In short, the good news is that if one averages (our observationally-based descriptions of) matter and radiation within the observable universe, on a sufficiently large spatial scale, then these observations support CP. To be a bit more precise about this, we need to respect the facts (as we scientific realists would say!) that the observable universe was once very small, that its matter inhomogeneities then were the seeds of later galaxies, and that (as I mentioned in (1) of Section 1.2) we cannot now directly observe (by any electromagnetic means) events earlier than the time of decoupling, about 400,000 years after the Big Bang. So to put it briefly: we should talk about sufficiently large spatial averaging at sufficiently late times after the Big Bang.

Indeed, this is not just a lucky break for present-day cosmology. We are all the more lucky for the CP having held up very well, over eighty years, as our astronomical and cosmological observations grew enormously: in the type of observation we could make (especially, covering non-optical wavelengths: radio, microwave, infra-red, ultra-violet, $\mathrm{X}$-ray), in their precision, and thereby in their spatiotemporal coverage or depth. To take a very simple example: we now know that there are tens of thousands of galaxies in each patch of the sky with a visual area equal to that of the full moon. Besides, over those 
eighty years, we have discovered some enormous cosmic structures: galaxies tend to be gathered in clusters separated by voids; and clusters themselves tend to be gathered in superclusters 10. Such clumping on very large length-scales obviously threatens the CP. However - so far as we know - there is no further clumping at length-scales yet larger than that of superclusters. And the observable universe is so vast as to contain thousands of superclusters: so that, while averaging over a typical length or volume of a supercluster, it still makes sense to ask whether the CP holds.

Here are some very approximate numbers. Let us ask: how large a scale must we consider in order to see no larger structure, i.e. so that we can ask whether the evidence on yet larger scales supports CP? Nowadays, the answer is: a vast scale, much vaster than was believed eighty years ago when the CP was formulated, i.e. before clusters and superclusters were found. Namely: we must consider scales of about 300 million light years or more. Yet this is still much smaller than the observable universe. Again let me put in numbers, albeit in a naive way.

Taking the universe to be now 10 billion $=10^{10}$ years old, our past light-cone has a linear spatial dimension at its base (i.e. soon after the Big Bang, but long enough after it so as to be well described by a standard FRW model, e.g. 400,000 years after) equal to 10 billion $=10^{10}$ light-years. As just mentioned, a spatial box on which CP is observed to be valid has a side-length $3 \times 10^{8}$ light years. So the ratio of side-lengths, between the base of our past light-cone and such a spatial box, is about: $3 \times 10$. So consider a vast cube, built of such boxes, standing on the square base of our past light-cone: a cube with side-length $10^{10}$ light years. This cube consists of a total of $(3 \times 10)^{3}=27 \times 10^{3} \approx 3 \times 10^{4}$ such boxes. These $3 \times 10^{4}$ boxes are a large enough set for it to make statistical sense to ask whether the CP holds.

Agreed, our past light-cone is a cone not a cube. That is: for many boxes at the base of the cube, the galaxies etc. in them have now moved through the boundary of our past light-cone. Once they are out, their light does not reach us; and in a universe that expands sufficiently rapidly, in particular one whose expansion accelerates, we would never receive the light (or other signals) emitted from such boxes. So these boxes are not sampled by any of our evidence (perhaps ever) from our past light-cone. But no worries: there are nevertheless thousands of boxes in our past light cone, so that we can ask what our evidence from observing these boxes suggests about the CP.

And luckily, one might say 'amazingly': many different kinds of evidence suggest that the CP does hold across all these boxes. (Agreed: (i) not as many as $3 \times 10^{4}$ boxes; cf. the concession in the previous paragraph; and (ii) only approximately; cf. the discussion of idealization at the start of Section 3.1.) The most famous evidence is of course the isotropy of the cosmic background radiation; (cf. (i) in footnote 7). But there are several other, largely independent, kinds of evidence, about optical, radio and X-ray sources. (For this evidence, Beisbart (2009, p. 186, 199) cites Kahav 2001, Sarkar et al (2009); I am

\footnotetext{
${ }^{10}$ Cf. e.g. Rowan-Robinson (2004, pp. 42-44, 113-119, 136-140), Longair (2006, pp. 253-268). For popular accounts of the discovery of clusters and superclusters, cf. Rowan-Robinson (1999, pp. 18-19, 52-54, 111-114), Silk (1989, pp. 192-245) and Smoot (1993, pp. 137-153). For full technical treatments of galaxy clustering, cf. Saslaw (1985, Part II; 2000).
} 
also grateful to W. Saslaw for pointing me to the most extensive recent discussion: Yang and Saslaw (2011).)

\subsection{Beyond the evidence: through a glass darkly}

Without wanting to take Saint Paul's name in vain, I cannot resist stealing his metaphor to describe cosmology's epistemic situation, in endeavouring to go beyond the evidence for the CP from within the past light cone of Earth-now; in Section 2] notation: the evidence from within $J^{-}$(Earth-now).

In this Subsection, I will first discuss some strategies for going beyond our causal past (Section 3.2.1). Then I will report doubts that have (rightly!) been raised about whether the CP holds. These doubts are based on considering inhomogeneities; and they question whether spacetime is homogeneous and isotropic (in the relevant sense, i.e. as regards matter, radiation and geometry, on sufficiently large scales) not just beyond, but also within, our past light-cone (Section 3.2.2) 11

\subsubsection{Strategies for justifying the CP}

I said in Section 3.1 .3 that the question whether the CP holds for the universe beyond our past light-cone can be addressed in various ways. As I see matters, there are three main ways. I will just note the first two strategies, and say more about the third strategy.

(1): Copernican?: First, there is the approach I noted at the start of Section 3.1.2. over the decades, various authors have adduced various rationalist grounds for believing the CP holds good for the whole universe. But I fully concur with the recent sceptical assessments of these grounds (by Beisbart and others, cited there). So I find this approach unpromising, and I would even say that it is over-emphasized in the philosophy of cosmology: so I set it aside.

(2): Inference?: The second way of addressing the issue is also somewhat 'aprioristic' (and so seems natural to philosophers). It is in play, but rarely explicit, in various discussions of the CP. Namely, one considers the various choices of spacetime region, beyond the observable universe $J^{-}$(Earth-now), that one might consider evidence about, or inference to; and then envisages that the evidence or inference will have different strengths or plausibilities for different regions. As one would expect, the considerations in play usually vary from one cosmological model (or family of models) to another, prohibiting a general discussion. But by way of illustration, here are three obvious specifications of spacetime regions one should consider:

(i): the causal past of our cosmic time-slice, i.e. $J^{-}(t=$ now) (for a model that has a cosmic time-function);

\footnotetext{
${ }^{11}$ So agreed: I could have reported these doubts in Section 3.1 instead of in this Subsection. But I think that would have been less clear. Anyway, placing them here means that the distinction between Section 3.1 and this Subsection is, not so much between our causal past and the rest of spacetime, as between the upbeat message in favour of the $\mathrm{CP}$ and the downbeat message against it - as I announced in Section 3 s preamble.
} 
(ii): the causal future of the observable universe, i.e. $J^{+}\left(J^{-}\right.$(Earth-now $\left.)\right)$;

(iii): the entire spacetime manifold, i.e. all spacetime points that are topologically connected to Earth-now 12

Mention of such regions prompts two salutary reminders about general methodology. First: in assessing evidence and making inferences, we must bear in mind our claims' implications for events in the unobservable regions, and conversely, how those events could constrain what we observe. (For a lucid introduction to these issues, cf. Ellis (1975)).

Second: although I noted in Section 3.1.3 how vast the observable universe is - how much vaster are regions such as (i) to (iii)! Just how vast they are is of course unknown, not least because estimates depend on the details of how we extend the standard cosmological model to times much earlier (logarithmically) than one second-extensions which are likely to long remain controversial (cf. Section 41). But to give an uncontroversial example, based on a 'late departure' from our past light cone: consider matter or radiation that left our past light cone at about the time of decoupling - as it might be, a cosmic background photon. That photon is estimated to now be about 40 billion light-years from us (the distance being measured along the cosmic time-slice $t=$ now). Vast indeed. This vastness emphasizes how risky is the induction from CP's holding in the observable universe (presumed on account of the evidence in Section 3.1.3) to its holding throughout any such region as (i), (ii) or (iii). To put the point very simply, in terms of enumerative induction over spacetime regions: the observable universe is such a small fraction of such regions, that it is risky to claim it is a fair sample 13

This riskiness is undoubtedly part of people's motivation in their search for general theoretical grounds for the CP holding throughout spacetime. I have set aside various rationalistic grounds, since I am sceptical of them. But there is a third way ...

(3): To be expected?: Third: Suppose we are given a cosmological theory, rather than a specific model, so that we envisage a range of possible initial conditions: where 'initial' need not mean 'at the Big Bang', but may mean only something like 'at times earlier than one second'. (So the theory could be some quantum field theory on a highly curved spacetime.) And suppose we could show that any, or any typical, initial condition at times earlier than one second evolves into a later state that is homogeneous and isotropicapproximately, i.e. on the sufficiently large scales that we now observe the CP holding. According to most physicists' and philosophers' views of explanation, such a demonstration would count as an explanation of the CP: indeed a satisfying one, that would be a great credit of the theory.

Note that in this situation, two familiar general ideas are in play: one philosophical, and one physical.

(i): The philosophical idea is that to explain is to show that the explanandum was to be expected: an idea with a tradition stretching from Aristotle to contemporary deductivenomological and causal accounts of explanation.

\footnotetext{
${ }^{12}$ Neither of regions (i) and (ii) includes the other; but of course region (iii) includes them both. And of course, other definitions are possible, and sometimes relevant.

${ }^{13}$ For more discussion of this risky induction, cf. Beisbart (2009, pp. 184-5, 199-201).
} 
(ii): The physical idea is a bit more specific, though also independent of theory. It is that initial conditions are a matter of mere happenstance, in some sense that a theory's laws (in particular: equations of motion) are not 14 So to show that a final state of affairs follows by the laws from an initial condition that is known, or reasonably believed, to have held, is - though informative, and perhaps even deserving of the name 'explanation' - not wholly satisfying. For if the initial condition invoked seems a matter of mere happenstance, then the demonstration in effect prompts the question: why did that initial condition hold?

Putting the two ideas together, one naturally concludes that an explanation (or at least: a satisfying explanation) of a final state of affairs must show, not just that it follows by the laws from an initial condition that is known, or reasonably believed, to have held, but also that it follows from any, or any typical, initial condition - and so was 'to be expected' in a stronger sense, independent of belief in a single initial condition.

Obviously, we are here at the threshold of several broad issues, which this paper can only mention, not pursue; though Section 4 will add a little.

(a): One issue is the history and philosophy of concepts of explanation, especially in relation to features of the universe as a whole. I recommend the work of McMullin (e.g. 1993, 2005, 2007): he calls the idea that typical (or even all) initial conditions lead to a final state with the feature of interest a 'Principle of Indifference'.

(b): A second issue is the idea of happenstance: can one make convincing precise sense of the idea that some particular propositions, such as initial conditions, are contingent in a stronger sense than are (according to we Humeans) the laws of nature? Note that one faces this issue, even if one is sceptical (as I am) that there is a single concept of explanation (or even: scientific explanation).

(c): Third: the CP, i.e. homogeneity and isotropy, is by no means the only feature of the 'late universe' for which the topic of the scope and limits of its explanation by invoking earlier states has been much discussed. Another main example is the direction of time, and its explanation in terms of an early 'smooth' equilibrium state 15 Section 4 will mention two other examples.

(d): Finally, a fourth issue arises from the fact that we can hardly expect to demonstrate that according to our cosmological theory, every initial condition evolves to a later state with the desired feature. So we must face the issue: what do we mean by a 'typical' initial condition? We can of course allow the answer to depend on the theory. In one theory, 'typical' might mean 'an open set in the topological space of initial conditions'; in another theory, it might mean 'with probability greater than 0.5 (or 0.9 ) according to some natural probability measure on the space of initial conditions'. But we must expect criticism and controversy about the physical, or explanatory, significance of the topology

\footnotetext{
${ }^{14}$ Two remarks. (i): This idea is of course compatible with the Humean view that laws are metaphysically and-or logically contingent: though the required stronger sense of 'mere happenstance' is rarely spelt out. (ii): I have stated the idea in terms of initial conditions: as is usual, and as suits our cosmological discussion. But one can, and some authors do, state the same idea for final and-or boundary conditions.

${ }^{15}$ For this example, recent philosophical discussion emphasizing the topic of explanation includes the debate between Callender (2004) and Price (2004) (the former downbeat, the latter upbeat, about how much one can ask of an explanation). For philosophical surveys that admirably clarify the physics involved, I recommend Callender (2011), Wallace (2010, 2011).
} 
or probability measure we have invoked.

Returning to our concern with justifying the CP: I note that two research programmesa past one, and a current one-have pursued this third ('To be expected') strategy for justifying the CP for the whole universe. The past programme is Misner's chaotic cosmology programme, the current one inflationary cosmology. Space prevents me going into any details; (though Section 4 will give a general summary of inflation). I recommend the following, which also emphasize philosophical issues: Barrow (1993, pp. 123-127), Barrow and Tipler (1986, pp. 420-430), Beisbart (2009, Section 5.1, pp. 190-193), Earman (1995, pp. 144-148, 152-155), Ellis (1999, pp. 706-713; 1999a, pp. 57-61; 2007, Sections 2.6 and 6.3, pp. 1208-1210, 1237-1238) and McMullin (2005, pp. 601-604; 2007, pp. 72-74); and for a popular account, Barrow (2011, pp. 161-168, 198-217).

So here it must suffice to sound two sceptical notes about these programmes, especially in relation to justifying the $\mathrm{CP}$.

(i): Misner's programme is generally regarded as having failed, for a variety of reasons (reviewed by e.g. Barrow (1993, pp. 123-124) and Earman (1995, pp. 144-145, 148)).

(ii): As to the inflationary programme, Section 4.3 will mention various reasons for scepticism about it. Here, I just follow Beisbart (2009, p. 192) in pointing out that although inflation prima facie seems to produce homogeneity by vastly stretching out inhomogeneities (cf. Section 4.1 for its solution to the flatness problem), this cannot really amount to justifying the CP. For inflationary cosmology envisages many stupendously distant regions of the entire universe that are typically - absent some fine-tuning of initial conditions, which would flout the spirit of the programme not approximately homogeneous.

\subsubsection{Inhomogeneities: doubts about the CP}

I close this Section by mentioning some doubts, based on considering inhomogeneities, about whether the CP holds not just beyond, but also within, our past light-cone. I will follow Ellis's recent survey of the issues (2011); (I also recommend the papers in the same issue which he cites).

So the over-arching question is whether the CP, or the FRW models it implies, is a justified large-scale description of the universe, despite the fact that it (and even its observable part) is evidently inhomogeneous in many ways and on many scales. Ellis distinguishes three main issues, and discusses them seriatim:

(1): inhomogeneities may affect the large-scale dynamics;

(2): inhomogeneities affect light propagation, and so may affect cosmological observations;

(3): maybe the universe is after all not spatially homogeneous on the largest scales and is better represented at late times by a Lemaitre-Tolman-Bondi spherically symmetric inhomogeneous model.

I shall set aside (2), but say a few words to explain (1) and (3).

(1): Inhomogeneities and dynamics:- The basic point is that in any dynamical system, coarse-graining in the sense of partitioning the space of states can fail to 'mesh' 
with the dynamics. That is: if $s_{1}, s_{2}$ are two states that the coarse-graining assigns to the same cell of the partition of the state-space (usually because $s_{1}, s_{2}$ match in their values for a select subset of quantities, usually called 'macro-quantities'), the later states into which $s_{1}, s_{2}$ evolve may well not be in the same cell. So at the level of the cells (the macro-quantities), there is no well-defined dynamics. Or in other words: the given dynamics is a well-defined 'micro-dynamics', but the coarse-graining does not properly define a 'macro-dynamics'.

This point is often made: sometimes (as by Ellis here) in the jargon that the dynamics does not commute with the coarse-graining. This matches the algebraic jargon, that a square diagram of functions (with arrows for time-evolution and for coarse-graining) can fail to be 'a commuting diagram'. Besides, even if there is commutation, the macrodynamics can look very different from the micro-dynamics. For introductory discussion and examples, cf. Butterfield (2011, Section 3, pp. 107-110), Ellis (2011b, Section 3.4.2).

Returning to our specific topic: here of course, we are given, not the micro-dynamics, which will be myriadly complex - even if, as is often done both in theory and in simulations, each large mass, say each galaxy, is treated as a point-particle. Rather, we are given a putative macro-dynamics, viz. the equations of general relativity, in a form that describes an expanding FRW model (and in order to describe time-evolution, cast in a ' $3+1$ ' form, rather than as Einstein's original field equations). And our question is whether this macro-dynamics is induced by a reasonable coarse-graining procedure on the unknown, myriadly complex, micro-dynamics. If we can be confident that it is thus induced, then the macro-dynamics, i.e. the FRW model, is indeed a justified large-scale description.

But evidently, this is a difficult question to answer! Indeed, even if we knew the complex micro-dynamics and coarse-graining procedure in full detail, it would probably be difficult to answer - for computational reasons. So it is perhaps unsurprising that Ellis goes on to survey no less than four substantially different, and still open, approaches to answering it; (2011, Section 2.3-2.5, pp. 4-9; cf. also Clarkson et al. 2011).

I should also mention what amounts to the 'converse' topic or question; (if only to distinguish it from our topic). It is often said that though the universe is expanding, gravitationally bound systems like the Earth or solar system are not expanding. In terms of the familiar pedagogic metaphor that the universe is like an inflating rubber balloon: such systems are like pennies glued to the balloon, with the glue being stiff enough that the rubber below a penny does not expand. Indeed, a vivid metaphor. But it prompts the questions: in the real universe, how large are the pennies - planets? solar systems? And what is the glue: i.e. the mechanism by which small enough systems are decoupled from the expansion? Thus this 'converse' topic is: the influence of the cosmological expansion on local dynamics. A wonderful topic, with a long history - and a masterly recent review by Carrera and Giulini (2010).

(3): Inhomogeneous models:- By and large, this paper has hitherto celebrated the achievements of the standard cosmological model, perhaps as modified by inflation (cf. Section 4); though (i) I have duly admitted the existence of respectable rivals ((b) at the end of Section 1.2), and (ii) this Subsection (Section 3.2) has had the downbeat message that we cannot now adequately justify the CP beyond the observable universe. In particular, I said in footnote 3 that the $\Lambda \mathrm{CDM}$ model is the best fit of the standard 
model to all cosmological observations we have made.

But now the cat is out of the bag! The point here is that the $\Lambda$ CDM model being the best fit of the standard model does not imply, of course, that it is the unique best fit model. And there is considerable evidence that the observations we have made so far can be equally well fitted by Lemaitre-Tolman-Bondi spherically symmetric inhomogeneous models - without, one might add, the all-too-conjectural dark energy of the $\Lambda$ CDM model. Indeed, this sort of rival to the standard model has a long history: apart from Ellis' recent introduction (2011, Section 4, pp. 11-16), cf. e.g. MacCallum (1979), Ellis (1991, pp. 563-564; 1999a, p.A60).

But again, I cannot pursue this topic. I just note that from a broad viewpoint, it represents 'business as usual', scientifically and philosophically. Scientifically, it may be possible, within the next ten to twenty years, to make observations that discriminate between the models. And meanwhile, philosophically: we have again illustrated the theme of cosmological under-determination: and more specifically, Section 3.2 s downbeat message that we cannot now adequately justify the CP.

\section{Pressing the question 'Why?'}

When one is given a description of anything, it is tempting, and perhaps inevitable, to press the question 'Why is that so?'. Besides, on most accounts of scientific explanation that distinguish description and explanation, one can legitimately press the 'Why?' question even when one is given an explanation. In particular, one can press it when given what this paper has celebrated: the established description of the observable universe from the time of primordial nucleosynthesis. Asking why the CP should hold (of the observable universe, or of the entire universe) forms one way of pressing the 'Why?' question in this context.

I will end this paper by briefly raising two other ways of pressing the question. Agreed: they lead to physical issues about times much earlier (logarithmically!) than one second after the Big Bang, and so to controversy: which in Section 1.1, I placed outside this paper's scope. But they relate so closely to justifying or explaining why the CP holds that some discussion, albeit brief, is in order.

Thirty years ago, these questions were dubbed 'the flatness problem' and 'the horizon problem'. Here 'problem' means 'problem for the then-current standard cosmological model (so incorporating the CP and its FRW metric)'. In brief: the problems were that this model stated that, but did not explain why:

(i) The universe at times later than about one second has an approximately flat (i.e. euclidean) spatial geometry: ('the flatness problem'; Section 4.1).

(ii) The photons in the cosmic background radiation that come to us along different directions in the sky have the same spectrum, even for directions different enough so that, according to this model, the regions on the last scattering surface, from which the photons were emitted 400,000 years after the Big Bang, at a temperature of about $4000 \mathrm{~K}$, could have had no previous causal contact with one another. This lack of contact means that there could not have earlier been any equilibration process by which the regions attained 
the same temperature: ('the horizon problem'; Section 4.2).

These explanatory lacunae were among the motivations for inflationary cosmological models - which apparently did explain (i) and (ii): namely, as arising from a conjectured exponential expansion during very early times, at about $10^{-35}$ seconds (e.g. Guth (1981)). The idea is that, given this exponential expansion:

(i): our entire universe arose from inflating a minuscule very early patch of spacetime, thus explaining its current approximate flatness;

(ii): the emission events for any two cosmic background photons, even two that come to us along opposite directions in the sky, do have a common past, i.e. their past light cones intersect; so that there could have been an earlier equilibration process making the temperatures of the emitting regions equal.

(Of course, the merits of inflationary models go well beyond these two explanations: in particular, they include predictions about the fluctuations in the photons' temperature, which have been confirmed by the COBE, WMAP and Planck projects-but I cannot discuss these here.)

Thus we return to the idea of explaining a feature of the 'late' universe by invoking conjectural earlier states, and to issues about the scope and limits of such explanations (cf. the third strategy, (3), of Section 3.2.1). In spelling out some details, we will glimpse (in Section 4.3) how inflationary cosmology's answer to these problems has led to proposals for, and much controversy about: both a multiverse ontology, and the idea of explaining features of the universe as arising from a selection effect (so-called 'anthropic explanations').

\subsection{The flatness problem}

Today's cosmologists favour inflationary cosmological models over the original standard models, on the grounds that they explain the value of the density parameter $\Omega$. In Newtonian terms, $\Omega$ is, roughly speaking, the ratio of the universe's gravitational potential energy to its kinetic energy. In general relativity, it is the ratio of the density of matter, radiation etc. to the density that would make the universe spatially flat, i.e. make the instantaneous spatial slices have asymptotically a euclidean geometry. So $\Omega$ is a pure dimensionless number. Observations show it to be very close to 1, i.e. its theoretically privileged value which makes for a flat universe. (For these details, cf. e.g. Liddle (2003, pp. 20, 48, 52-55, 132); $\Omega=1$ corresponds to $k=0$ in (ii: a) of Section [3.1.1.)

The original standard models treated the value of $\Omega$ as a brute fact, in the sense of being a fact that is set aside from the quest for explanation. Agreed, it is no doubt a matter of judgment which facts it is legitimate to treat as brute; and this is a judgment that often varies from one context or episode of enquiry to another, and which may be contentious or even subjective. An oft-cited historical example of this sort of variation is Kepler's invoking a nested sequence of the Platonic solids, snugly fitting into each other like Chinese boxes, to explain the relative sizes of the orbits of the planets: but this is a fact which Newtonian celestial mechanics later came to treat as brute 16 So for all that

\footnotetext{
${ }^{16}$ This example also illustrates how strongly, though unconsciously, education inculcates a sense of
} 
I have so far said, one might well conclude that the original Big Bang/standard models treating $\Omega$ as a brute fact was no discredit to them.

But it seems unsatisfying to have nothing to say about why a parameter is close to its theoretically privileged value. And it is all the more worrying if being close to this value now requires being much closer to it, in the past - as happens in this case: for $\Omega$ to be about 1 today required that it be extraordinarily close to 1 very soon after the Big Bang. For example: according to a simple model, assuming merely that $\mid \Omega($ now $)-1 \mid<0.5$ implies that $\Omega$ has to be $1 \pm 10^{-18}$ one second after the Big Bang, and $1 \pm 10^{-30}$ at $10^{-12}$ seconds after it. (This is because in this model $|\Omega(t)-1| \propto t$, and the present age of the universe is about $4 \times 10^{17}$ seconds; cf. e.g. Liddle (2003, p. 100).) I think the natural reaction to so exquisite a tuning of a dimensionless number to its privileged value is that it calls out for explanation: we should not treat the observed value as a brute fact.

In any case, this has been the reaction of cosmologists - at least, after the inflationary models were devised, so that one had a putative explanation to assess and perhaps endorse. Besides, the basic idea of this explanation was attractively simple, and the same for the various inflationary models. Roughly speaking, the idea is that any manifold 'looks flat' on a sufficiently small scale. More formally: about any point in a Riemannian manifold, there is a neighbourhood that is sufficiently small that its metric structure is as close as you please to being euclidean. Transferring this idea to the context of an expanding spacetime, we infer that if our entire universe arose from a suitably fast, say exponential, expansion of a minuscule very early patch of spacetime, its spatial slices would have, after this expansion, an approximately euclidean geometry. A bit more specifically, in terms of inflationary cosmology: $\Omega$ will be driven very close to 1 by the end of the inflation period. (For some details, cf. Lawrie (1990, pp. 333-334), Liddle (2003, p. 104-107).)

\subsection{The horizon problem}

As emphasized above (especially Section 3.1.3), the cosmic background radiation, dating from the decoupling time $t_{d}$ (400,000 years after the Big Bang), is to a very good approximation isotropic. This means that all the emitting regions were at about the same temperature. But for two directions in space with a sufficient angular separation, the two past events at the time $t_{d}$ that lie along those directions, i.e. the two events of emitting a photon that we receive along that direction, had-according to the standard Big Bang models - no common causal past. That is, their past light cones did not intersect. Calling the events $p, q \in M$, we have: $J^{-}(p) \cap J^{-}(q)=\emptyset$. This causal separation means that no equilibration process (or any other sort of interaction) could have occurred earlier, so as to establish the emitting regions' almost equal temperatures.

Similarly to the flatness problem, one might at first think that this is curious, but not an intellectual problem. That is, one might think that the standard models' thus needing an early state (at least as early as $t_{d}$ ) to be special, in that it encodes these equal

which facts should be treated as brute: anyone learning Newtonian mechanics, yet unaware of Kepler's struggles, would never think to ask why the planets' orbits are the sizes that they are. We are taught to treat those sizes as 'matters of mere happenstance'; (cf. the discussion in (3) (ii) of Section 3.2.1). 
temperatures, is no discredit to them. Why should we hope that the features of early states can be explained in some strong sense, for example by exhibiting them as generic? Of course, the notions of 'explanation' and 'generic' in play here would need to be clarified: cf. Section 3.2.1s discussion of its third strategy, (3). But the idea will be: the most we can reasonably hope for is that they are retrodicted by our theory's description of later states (which we will no doubt have to describe collectively, rather than individually, to get enough information for a retrodiction): 'the early states were what led to this - end of story, 17

But I think that the quantitative details show that there is indeed an intellectual problem, or at least 'embarrassment'. (Again, I think this is analogous to the flatness problem: there, it was the quantitative exquisiteness of the tuning of $\Omega$ that called out for explanation.) I have in mind not just the nearness of the equality of the temperatures, for all directions in the sky: namely, equality to about one part in $10^{5}$. Also, and perhaps more striking: the directions in the sky for which, according to the standard models, the photon emission-events have no common causal past, can be very close. You might expect that they need to be approximately perpendicular, or even opposite, to each other, for there to be the 'embarrassment' of same temperature with no possible explanation (by the standard models) invoking previous interaction. But no: the directions can be separated by as little as one or two degrees; (cf. e.g. Liddle (2003, pp. 102, 109), Lawrie (1990, p. 326-7). Since there are 360 degrees in a circle, this means that according to the standard models, the last scattering surface is composed of tens of thousands of regions, any pair of which has no common causal past - yet all 'embarrassingly' at the same temperature, to about one part in $10^{5}$.

Besides, the number of causally disconnected regions grows embarrassingly steeply, as one runs the standard model further back in time than $t_{d}$. Barrow (1993, p. 126; 2011, p. 205) gives a simple estimate: at $t=10^{-35}$ seconds, the observable universe would have been about $1 \mathrm{~cm}$ across, and a causally connected region would have been about $10^{-24}$ $\mathrm{cm}$ across. So, thinking naively of these regions as forming a cube: there would then have been $\left(10^{24}\right)^{3}=10^{72}$ such regions, all mutually causally disconnected.

In any case, again analogously to the flatness problem: the cosmologists' verdict has been that there is a problem - at least, after the inflationary models were devised, so that one had a putative explanation to assess and perhaps endorse. And again, the basic idea of this explanation was attractively simple, and the same for the various models: the

\footnotetext{
${ }^{17}$ This irenic response can be filled out somewhat, using our notation $J^{-}$for causal pasts, in the context of a future-deterministic relativistic theory - such as we would expect a classical cosmology to be. (This point is made by Earman (1995, pp. 139-140), in the course of his masterly discussion (ibid., Chapter 5) of the horizon problem.) In such a theory, the state on a hypersurface $\Sigma$ that extends across a spacetime region $R$ determines the state throughout the future domain of dependence $D^{+}(\Sigma \cap R)$. (The future domain of dependence $D^{+}(Q)$ of any region $Q$ is defined as the set of spacetime points through which any past-directed causal (everywhere timelike or lightlike) curve must intersect Q. Intuitively, it is the set of points on whom any causal influence must first register on $Q$.) So if we (i) pick the region $R$ to be $J^{-}(p) \cup J^{-}(q)$, and (ii) take $\Sigma$ to extend across $R$ prior to the 'summit' of $J^{-}(p) \cap J^{-}(q)$, and (iii) consider as an initial condition (or as a premise of a deductive-nomological explanation) the state on $\Sigma \cap\left(J^{-}(p) \cup J^{-}(q)\right)$ : then we can surely give a prediction, and so surely an explanation, of the states at $p$ and at $q$. As the song says: 'who could ask for anything more?'
} 
inflation period implies that the events $p, q$ have a common causal past - even for two opposite directions in the sky. That is: $J^{-}(p) \cap J^{-}(q) \neq \emptyset$, so that there could have been an earlier process of equilibration of the desired kind.

Finally, I should mention a connection with a worthy perennial of the philosophy of probability and causation, as well as of quantum non-locality: Reichenbach's Principle of the Common Cause (PCC: 1956, Section 19). Roughly speaking, the PCC says that if two events are correlated, but neither causes the other, then in their common causal past there is a third event, conditional on which they are probabilistically independent (called the 'common cause' or 'screener-off') . Notwithstanding this abstract formulation, countless examples in everyday life and the sciences exemplify the principle, making it intuitively plausible. And clearly, the 'embarrassment' that constitutes the horizon problem echoes this plausibility: since the two emission-events' temperatures are so well correlated, surely some third event in their common causal past influenced them both 18

\subsection{Questions beyond}

So much by way of motivating the idea of inflation. So far, the broad methodological situation seems as described in the third approach, (3), of Section 3.2.1. Namely: one buys a satisfying explanation of a 'late' feature of the universe, by paying the price of a speculative piece of physics for very early times.

But this is not to suggest that the situation is simple, or settled. Apart from the broad issues about explanation etc. mentioned in (3) of Section 3.2.1, there are many physical details relevant to the methodological issues: for example, about these models assuming a wholly conjectural inflaton field, about there being embarrassingly many such inflation models, and about how these models are meant to secure that the inflation period ends. (These issues are pressed by, e.g. Ellis (1999a, p. A59; 2007, Section 5, pp. 1232-1234), Earman and Mosterin (1999).)

Besides, some of these details raise much wilder ideas, in particular the multiverse: which, by way of conclusion, I briefly report. (For details, cf, e.g. Albrecht (2004), Linde $(1987,1990,2004)$.)

(1): First, the idea of inflation leads to require models in which, during the inflation

\footnotetext{
${ }^{18}$ Hofer-Szabo et al. (2013) is a definitive monograph on the PCC. My own view of its role as a motivation for the locality assumptions in Bell's theorem is in Butterfield (2007). Here it must suffice to note a contrast between quantum non-locality and inflationary cosmology. (1): As Hofer-Szabo et al. emphasize, Bell's theorem needs to assume, not only that the two events in each of several pairs have a common cause (in the sense of rendering the two events probabilistically independent), but also that one and the same event is the common cause for the several pairs (and so is called a "common common cause'): an assumption which Nature apparently violates. (2): In inflationary cosmology, the physical situation is of course both much more complicated and much more conjectural. But the idea is that the photon-temperature correlations observed between many, if not all, pairs of directions in the sky are screened off by a single, suitably extended, event or process of equilibration in the pairs' common past. So using Hofer-Szabo et al.'s jargon, the idea is that there is a common common cause: an assertion which - if we judge by the recent evidence favouring inflation-Nature obeys. Finally, I should also note that the maestro Earman takes a broadly sceptical view of the significance of the PCC, and indeed of the 'embarrassment'; (1995, pp. 135-140, 142-146, 156; Earman and Mosterin (1999)).
} 
period, countless spacetime regions branch off and themselves inflate to yield a 'baby universe'. Since what we have so far called 'the entire universe' (or in other words: 'a standard cosmological model') would correspond to one such region ('our region'), the totality of regions is dubbed 'the multiverse'. (But 'many-headed Hydra' would be a more vivid name.)

(2): Besides (though I have not yet admitted it): inflationary models are quantum theoretic, so that the events of a region branching off, and of its inflating, and the details of how the branching and expansion occur, are all stochastic events, with probabilities given by quantum theory.

(3): Furthermore, the idea of inflation requires models in which, generically, the stochastic process of growing branches goes on forever. Hence the slogan that 'inflation is generically eternal'.

(4): And finally: some inflationary models assume that the prevailing laws of physics, in particular the values of physical constants (such as the charge of the electron, or the ratio of the strengths of the gravitational and electromagnetic forces), vary across the different regions. To take a simple example: consider a region whose state just after its inflation period matches our own region's state at the corresponding time, i.e. just after our inflation period, except that this region has a gravitational force a little stronger than ours. That force would soon halt the region's post-inflation expansion, and lead to collapse, called a 'Big Crunch' on analogy with 'Big Bang'. (For numerical estimates for 'a little stronger' and 'soon', cf. e.g. Dicke and Peebles (1979, p. 514).)

To sum up: the totality of all these spacetime regions is a stupendous, and in general infinite, probabilistic branching structure. The regions are linked together by narrow throats, rather like the elongated bubbles in the balloon sculptures of dogs or what-not made by magicians and street performers. And according to some models, the laws of physics, in particular the values of physical constants, vary across the regions. Obviously, we here glimpse many undoubtedly wild questions: both physical and philosophicalabout what should count as explaining initial conditions or the values of constants, about selection effects, ontological parsimony etc. Opinion is strongly divided about such questions, and I must postpone discussion of them to another place 19

Acknowledgments: This paper is dedicated to the memory of Ernan McMullin, whose contributions to the philosophy of science were so wise, insightful and scholarly; and whose energetic and generous spirit enhanced so many lives. For correspondence and comments on previous versions, I am very grateful to the editor and two anonymous referees; and to Nazim Bouatta, Claus Beisbart, George Ellis, John Manchak, John Norton, Brian Pitts, Chris Smeenk, Arianne Shahvisi, Bill Saslaw, David Sloan, Bill Stoeger, Henrik Zinker-

\footnotetext{
${ }^{19}$ For maestri being sceptical about inflation, I recommend: Ellis (1999, p. 706-707; 1999a, pp. A59, A64-65; 2007, Section 5, pp. 1232-1234), Earman (1995, pp. 149-159), Earman and Mosterin (1999), Penrose (2004, pp. 735-757, Chapters 28.1-28.5) and Steinhardt (2011). For philosophical discussion of the multiverse and selection effects, I recommend: Ellis (1999, pp. 696-700, 707-712; 2011a), Smeenk (2012, Sections 6 to 8), Wilzcek (2007) and the other papers in Carr (2007), and Zinkernagel (2011, Section 4.1).
} 
nagel, Lena Zuchowski. I also thank John Barrow, George Ellis and Henrik Zinkernagel for encouragement, and for the inspiration of their writings. Work on this paper was supported by a grant from the Templeton World Charity Foundation, which I gratefully acknowledge.

\section{References}

Albrecht, A. (2004), 'Cosmic inflation and the arrow of time', in J. Barrow, P. Davies and C. Harper eds., Science and Ultimate Reality: quantum theory, cosmology and complexity, Cambridge University Press, pp. 363-401.

Balashov, Y. (1994), 'Uniformitarianism in cosmology: background and philosophical implications of the steady-state theory', Studies in History and Philosophy of Science 25, number 6, pp. 933-958.

Balashov, Y. (2009), 'A cognizable universe: transcendental arguments in physical cosmology', in ed. M. Bitbol et al. Constituting Objectivity, Springer, pp. 269-278.

Barrow, J. (1993), 'Unprincipled cosmology', Quarterly Journal of the Royal Astronomical Society 34, pp. 117-134.

Barrow, J. (2011), The Book of Universes, London: Bodley Head.

Barrow, J. and Tipler, F. (1988), The Anthropic Cosmological Principle, Oxford University Press.

Beisbart, C. (2009), 'Can we justifiably assume the Cosmological Principle in order to break model under-determination in cosmology?', Journal of General Philosophy of Science 40, pp. 175-205.

Beisbart, C. (2012), 'The many faces of the Cosmological Principle', in preparation.

Beisbart, C. and Jung, T. (2006), 'Privileged, typical or not even that? Our place in the world according to the Copernican and cosmological principles', Journal of General Philosophy of Science 37, pp. 225-256.

Butterfield, J. (2007), 'Stochastic Einstein Locality Revisited', British Journal for Philosophy of Science 58, pp. 805-867.

Butterfield, J. (2011), 'Laws, causation and dynamics at different levels', Interface Focus (The Royal Society, London) 2, pp. pp. 101-114.

Butterfield, J. (2012), 'Under-determination in cosmology: an invitation', The Aristotelian Society Supplementary Volume 2012, 86, pp. 1-18.

Callender, C. (2004), 'Measures, explanation and the past: should 'special' initial conditions be explained?', British Journal for the Philosophy of Science 55, pp. 195-217.

Callender, C. (2011), 'Hot and heavy matters in the foundations of statistical mechanics', Foundations of Physics 41, pp. 960-981. 
Carr, B. (2007), Universe or Multiverse?, Cambridge University Press.

Carrera M. and Giulini, D. (2010), 'Influence of global cosmological expansion on local dynamics and kinematics', Reviews of Modern Physics 82, pp. 169-208.

Clarkson, C., Ellis, G., Larena, J. and Umeh, O. (2011), 'Does the growth of structure affect our dynamical models of the universe?', arxiv: astro-ph:1109.2314

Clifton, T., Clarkson C. and Bull P. (2011), 'The isotropic blackbody CMB as evidence for a homogeneous universe', arxiv: 1111.3794

Dicke, R. and Peebles, J. (1979), 'The Big Bang cosmology - enigmas and nostrums', in S. Hawking and W. Israel eds., General Relativity: an Einstein Centenary Survey, Cambridge University Press; pp. 504-517.

Earman, J. (1995), Bangs, Crunches, Whimpers and Shrieks, Oxford University Press.

Earman, J. and Mosterin, J (1999), 'A critical look at inflationary cosmology', Philosophy of Science 66, pp. 1-49.

Ehlers, J., Geren P., and Sachs R. (1968), 'Isotropic solutions of the Einstein-Liouville equations', Journal of Mathematical Physics 9, pp. 1344-1349.

Ellis, G. (1975), 'Cosmology and verifiability', Quarterly Journal of the Royal Astronomical Society, 16, pp. 245-264.

Ellis, G. (1991), 'Major themes in the relation between philosophy and cosmology', Memorie della Societa Astronomica Italiana 62, pp. 553-605.

Ellis, G. (1993), 'The physics and geometry of the universe: changing viewpoints', Quarterly Journal of the Royal Astronomical Society 34, pp. 315-330.

Ellis, G. (1999), 'Before the beginning: emerging questions and uncertainties', Astrophysics and Space Science 269-270, pp. 693-720.

Ellis, G. (1999a), '83 years in general relativity and cosmology: progress and problems', Classical and Quantum Gravity 16, pp. A37-A75.

Ellis, G. (2007), 'Issues in Philosophy of Cosmology', in part B of J. Butterfield and J. Earman eds, Philosophy of Physics, Elsevier, volume 2 of the North Holland series, The Handbook of Philosophy of Science, pp. 1183-1286; astro-ph/0602280.

Ellis, G. (2011), 'Inhomogeneity effects in cosmology', Classical and Quantum Gravity 28, 164001.

Ellis, G. (2011a), 'Does the multiverse really exist?', Scientific American 305, August 2011, pp. 38-43.

Ellis, G. (2011b), 'On the limits of quantum theory: contextuality and the quantumclassical cut', arxiv: quant-ph:1108.5261

Ellis, G., S. Nel, R. Maartens, W. Stoeger and A. Whitman (1985), 'Ideal observational cosmology', Physics Reports 124, pp. 315-417.

Glymour, D. (1977), 'Indistinguishable spacetimes and the fundamental group', in J. Earman, C. Glymour and J. Stachel (ed.s), Foundations of Spacetime Theories, Minnesota 
Studies in Philosophy of Science volume 8, University of Minnesota Press, pp. 50-60.

Guth, A. (1981), 'Inflationary universe: a possible solution to the horizon and flatness problems', Physical Review D 23, pp. 347-356.

Hawking, S. and Ellis, G. (1973), The Large-scale Structure of Spacetime, Cambridge University Press.

Hofer-Szabo, G., M. Redei and L. Szabo (2013), The Principle of the Common Cause, forthcoming, Cambridge University Press.

Kolb, E. and Turner, M. (1990), The Early Universe, Westview Press: Frontiers in Physics.

Kragh, H. (1996), Cosmology and Controversy, Princeton University Press.

Lahav, O. (2001), 'Observational tests for the cosmological principle and world models', in R. Crittenden adn N. Turok (eds), NATO ASIC Proc 565: Structure Formation in the Universe, pp. 131f.

Lawrie, I. (1990), A Unified Grand Tour of Theoretical Physics, Institute of Physics Publishing, Adam Hilger.

Liddle, A. (2003), An Introduction to Modern Cosmology, John Wiley; second edition.

Linde, A. (1987), 'Inflation and quantum cosmology', in S. Hawking and W. Israel eds., 300 Years of Gravitation, Cambridge University Press; pp. 604-???.

Linde, A. (1990), Particle Physics and Inflationary Cosmology, Harwood Academic.

Linde, A. (2004), 'Inflation, quantum cosmology and the anthropic principle', in J. Barrow, P. Davies and C. Harper eds., Science and Ultimate Reality: quantum theory, cosmology and complexity, Cambridge University Press, pp. 426-458.

Longair, M. (2003), Theoretical Concepts in Physics, Cambridge University Press; second edition (first edition 1984).

Longair, M. (2006), Cosmic Century, Cambridge University Press.

MacCallum, M. (1979), 'Anisotropic and inhomogeneous relativistic cosmologies', in S. Hawking and W. Israel eds., General Relativity: an Einstein Centenary Survey, Cambridge University Press; pp. 533-580.

Magnus, P. (2005), 'Reckoning the shape of everything: underdetermination and cosmotopology', British Journal for the Philosophy of Science 56, pp. 541-557.

Malament, D. (1977), 'Observationally indistinguishable spacetimes', in J. Earman, C. Glymour and J. Stachel (ed.s), Foundations of Spacetime Theories, Minnesota Studies in Philosophy of Science volume 8, University of Minnesota Press, pp. 61-80.

Manchak, J. (2009), 'Can we know the global structure of spacetime?', Studies in History and Philosophy of Modern Physics 40, pp. 53-56.

Manchak, J. (2011), 'What is a physically reasonable spacetime?', Philosophy of Science 78, pp. 410-420.

McMullin, E. (1985), 'Galilean idealization', Studies in the History and Philosophy of 
Science 16, pp. 247-273.

McMullin, E. (1993), 'Indifference principle and anthropic principle in cosmology', Studies in the History and Philosophy of Science 24, pp. 359-389.

McMullin, E. (2005), 'Anthropic explanation in cosmology', Faith and Philosophy 22, pp. 601-614.

McMullin, E. (2007), 'Tuning fine-tuning', in J. Barrow et al. eds., Fitness of the Cosmos for Life: Biochemistry and fine-tuning, Cambridge University Press; pp. 70-94.

Misner, C., Thorne K. and Wheeler, J (1973), Gravitation, W.H. Freeman.

Nadathur, S., Hotchkiss, S. and Sarkar, S. (2011), 'The integrated Sachs-Wolfe imprints of cosmic superstructures: a problem for $\Lambda$ CDM', http://arxiv.org/abs/1109.4126v1.

Norton, J. (2011), 'Observationally indistinguishable spacetimes: a challenge for any inductivist', In G. Morgan, ed., Philosophy of Science Matters: the Philosophy of Peter Achinstein Oxford University Press, 2011, pp. 164-176. Available at:

http://www.pitt.edu/ jjdnorton/papers/Obs_Equiv_final.pdf

Penrose, R. (2004), The Road to Reality, Jonathan Cape.

Price, H. (2004), 'On the origins of the arrow of time: why there is still a puzzle about the low entropy past', in C. Hitchcock ed. Contemporary Debates in Philosophy of Science, Oxford; Blackwell, pp. 219-239.

Reichenbach. H. (1956), The Direction of Time, University of California Press.

Rees, M. (1997), Before the Beginning, Simon and Schuster.

Rees, M. (2003), 'Our complex cosmos and its future', in G. Gibbons, E. Shellard and S. Rankin (ed.s), The Future of Theoretical Physics and Cosmology, Cambridge University Press, pp. 17-37.

Rindler, W. (1977), Essential Relativity, Springer: second edition. Press.

Rowan-Robinson, M. (1999), The Nine Numbers of the Cosmos, Oxford University

Rowan-Robinson, M. (2004), Cosmology, Oxford University Press: fourth edition.

Rugh, S. and Zinkernagel, H. (2009), 'On the physical basis of cosmic time', Studies in History and Philosophy of Modern Physics 40, 1-19.

Rugh, S. and Zinkernagel, H. (2011), 'Weyl's principle, cosmic time and quantum fundamentalism', in D. Dieks et al. (eds.), Explanation, Prediction and Confirmation: the philosophy of science in a European perspective, Springer, pp. 411-424.

Sarkar, S. (2007), 'Is the evidence for dark energy secure?, http://arxiv.org/abs/0710.5307v2

Sarkar, P. et al (2009), 'The scale of homogeneity of the galaxy distribution in SDSS DR6', Monthly Notices of the Royal Astronomical Society 399, pp. L128-L131.

Saslaw, W. (1985), Gravitational Physics of Stellar and Galactic Systems, Cambridge University Press.

Saslaw, W. (2000), The Distribution of the Galaxies, Cambridge University Press. 
Sciama, D. (1971), Modern Cosmology, Cambridge University Press.

Silk, J. (1989), The Big Bang, W.H. Freeman; revised and updated edition.

Silk, J. (2006), The Infinite Cosmos, Oxford University Press.

Smeenk, C. (2012), 'Philosophy of cosmology', in The Oxford Handbook of Philosophy of Physics, ed. R. Batterman, Oxford University Press: pp. 607-652.

Smoot, G. (1993), Wrinkles in Time: the imprint of creation; (written with Keay Davidson), Little Brown and Company.

Steinhardt, P. (2011), 'The inflation debate', Scientific American 304, April 2011, pp. $38-43$.

Stoeger, W., Maartens, R., and Ellis, G. (1995), 'Proving almost-homogeneity of the universe: an almost Ehlers, Geren and Sachs theorem', Astrophysical Journal 443, pp. $1-5$.

Torretti, R. (1983), Relativity and Geometry, Pergamon Press: reprinted by Dover.

Wald, R. (1984), General Relativity, University of Chicago Press.

Wallace, D. (2010), 'Gravity, entropy and cosmology: in search of clarity', British Journal for the Philosophy of Science 61, pp. 513-540.

Wallace, D. (2011), 'The logic of the past hypothesis', forthcoming; available at: http://philsci-archive.pitt.edu/8894/

Weinberg, S. (1972), Gravitation and Cosmology, New York: John Wiley.

Weinberg, S. (2008), Cosmology, Oxford University Press.

Wilzcek, F. (2007), 'Enlightenment, knowledge, ignorance, temptation', in B.Carr (ed.), Universe or Multiverse?, Cambridge University Press, pp. 43-54. arXiv: hep-ph/0512187v2

Yang, A. and Saslaw, W. (2011), 'The galaxy counts-in-cells distribution from the SDSS', Astrophysical Journal 729, 123: arXiv:1009.0013v2. doi:10.1088/0004-637X/729/2/123

Zinkernagel, H. (2002), 'Cosmology, particles and the unity of science', Studies in History and Philosophy of Modern Physics 33B, pp. 493-516.

Zinkernagel, H. (2011), 'Some trends in the philosophy of physics', Theoria (Spain) 26, pp. 215-241; available at: http://philsci-archive.pitt.edu/8761/ 